\title{
Essais
}

ESSAIS

Revue interdisciplinaire d'Humanités

10 | 2016

Faire-valoir et seconds couteaux

\section{Confusion à la Maison Blanche : la bataille du pouvoir entre Hillary Clinton et Al Gore}

Pierre-Marie Loizeau

\section{OpenEdition}

1 Journals

Édition électronique

URL : http://journals.openedition.org/essais/4002

DOI : 10.4000/essais.4002

ISSN : 2276-0970

Éditeur

École doctorale Montaigne Humanités

Édition imprimée

Date de publication : 15 septembre 2016

Pagination : 115-129

ISBN : 978-2-9544269-9-0

ISSN : 2417-4211

Référence électronique

Pierre-Marie Loizeau, « Confusion à la Maison Blanche : la bataille du pouvoir entre Hillary Clinton et Al Gore », Essais [En ligne], 10 | 2016, mis en ligne le 15 octobre 2020, consulté le 26 octobre 2020. URL : http://journals.openedition.org/essais/4002 ; DOI : https://doi.org/10.4000/essais.4002 


\title{
Confusion à la Maison Blanche : la bataille du pouvoir entre Hillary Clinton et Al Gore
}

\author{
Pierre-Marie Loizeau
}

La présidence Clinton, qui s'est étalée sur deux mandats successifs (19932001), a déjà fait l'objet de nombreuses études ayant mis en relief les problèmes économiques, les enjeux sociaux et culturels, et les défis internationaux auxquels elle fut confrontée. Comme pour chaque administration, éditorialistes, historiens et politologues ont mené une réflexion sur le pouvoir et l'art de gouverner face aux convulsions de l'histoire contemporaine. Cependant, rares sont les recherches ayant mis en avant les rivalités internes au sein de l'équipe présidentielle durant ces huit années. Or, celles-ci furent bien réelles. Elles apparurent dès le début du premier mandat, au sortir d'une campagne électorale intense qui laissait déjà entrevoir un télescopage des futures responsabilités dans le proche entourage du candidat. Si la capacité de « leadership » de ce dernier ne souffrait d'aucune ambiguïté, c'est l'omniprésence de son épouse Hillary Clinton dans les lieux de pouvoir qui entraîna une redistribution des rôles et jeta le trouble dans la relation traditionnelle entre le président et son vice-président.

Nous chercherons donc dans un premier temps à déterminer les fonctions et le positionnement du vice-président et de la First Lady au sein de la sphère présidentielle et à analyser ensuite comment et jusqu’à quel point la rivalité entre ces deux personnages-clé a pu entraver l'exercice même du pouvoir et éroder la crédibilité du gouvernement Clinton.

\section{Vice-présidence : une fonction superflue?}

Historiquement, la vice-présidence n’a joué qu'un rôle très limité voire symbolique dans la conduite de la nation. À l'origine, cette fonction fut créée essentiellement pour écarter toute éventualité d'absence de président en cas de décès, démission, destitution ou encore incapacité physique ou mentale à exercer le pouvoir. Les auteurs de la Constitution empêchèrent ainsi toute possibilité de voir la nation sans exécutif, sachant que dans le système de gouvernement qu'ils venaient de mettre en place, prenant soin de séparer et 
répartir équitablement les pouvoirs, il était impossible de se passer d'un "chief executive ". Le premier vice-président à ainsi accéder à la Maison Blanche, suite au décès du président, fut John Tyler en 1841 en remplacement de William Henry Harrison. ${ }^{1}$ En revanche, s'il ne peut y avoir de vacance du pouvoir présidentiel, le pays s'est bel et bien passé de vice-président à plusieurs reprises, la période la plus longue étant celle de 1850 à 1857 (969 jours) sous la présidence Fillmore.

Les Pères Fondateurs réunis à Philadelphie (25 mai-17 septembre 1787) doutèrent de l'utilité d'un vice-président. Comme l'écrit Ray Raphael, "the vice-president was the bastard son of the convention, which knew not how to deal with him. $»^{2}$ Il fallut un compromis de "dernière minute " pour inclure le poste dans la Constitution. La seule véritable fonction explicite qui fut alors mentionnée fut celle de président du Sénat. Ainsi George Mason, le père du "Bill of Rights ", fustigea-t-il " the unnecessary office of the Vice President, who for want of other employment is made president of the Senate, thereby dangerously blending the executive and legislative branches. ${ }^{3}$ John Adams, le premier vice-président de l'histoire, fut lui-même très critique sur sa fonction : "My country has in its wisdom contrived for me the most insignificant office that ever the invention of man contrived or his imagination conceived. $»^{4}$

La ratification du XII ${ }^{e}$ amendement apporta une évolution majeure au mode d'élection. Le précédent système, tel que le décrit la Constitution à l'origine, précisait que chaque grand électeur devait voter pour deux personnes à la fois. Le candidat ayant reçu le plus de voix était alors élu président et le second candidat le mieux placé était élu vice-président. Selon l'amendement de 1804, afin d'éviter que ne se reproduise le cas de figure de 1800 où les deux candidats Thomas Jefferson et Aaron Burr avaient obtenu le même nombre de voix, le Congrès requit un vote séparé pour le président et le vice-président.

"The Electors shall meet in their respective states, and vote by ballot for President and Vice-President (...); they shall name in their ballots the person voted for as President, and in distinct ballots the person voted for as VicePresident, and they shall make distinct lists of all persons voted for as President, and all persons voted for as Vice-President (...) $»^{5}$

1 Le libellé très ambigu de la Constitution fut sujet à une grande controverse concernant la succession de Harrison. John Tyler créa en effet un précédent, le fameux "Tyler precedent ", en refusant le statut de simple " acting president » et en se déclarant président à part entière.

2 Raphael Ray, Mr. President - How and Why the Founders Created a Chief Executive (New York: Knopf, 2012), p. 120.

3 Ibid., p. 130.

4 Waldrup Carole Chandler, The Vice Presidents (Jefferson, N.C.: McFarland \& Company, Inc., Publishers, 1996), p. viii.

5 "The Constitution of the United States of America ", Amendment XII. <http://constitutioncenter.org/constitution/the-amendments/amendment-12-choosing-the-president-vice-president> Consulté le 13 juin 2015. 
Ce mode de nomination permet en effet de donner plus de cohérence au processus et plus de cohésion au "ticket ", mais fatalement il ne laisse guère de suspense et fait du " running mate " un simple appendice ou faire-valoir du candidat présidentiel. Comme le regretta le Représentant du Massachusetts Samuel Taggart pendant les débats : " the vice president will not stand on such high ground in the method proposed as he does in the present mode of a double ballot " et ajouta " great care will not be taken in the selection of a character to fill that office. ${ }^{6}$ Faible constitutionnellement depuis son origine et désormais dépourvue de prestige politique, la fonction n'attira guère les hommes de talent. "I do not propose to be buried before I am dead ", déclara Daniel Webster, le leader du parti Whig quand on lui proposa le poste en 1848.7 Parmi les vice-présidents du XIX siècle, figurent d'ailleurs des hommes politiques de moindre envergure ou intégrité. Citons par exemple Schuyler Colfax et Henry Wilson (sous Grant) qui furent impliqués dans le célèbre scandale du Crédit Mobilier dans les années 1870. ${ }^{8}$

Le début du XX $\mathrm{X}^{\mathrm{e}}$ siècle amorça une nouvelle ère et des évolutions marquantes. C'est Theodore Roosevelt, élu en 1900, qui fut le grand promoteur de la viceprésidence en multipliant les voyages et autres déplacements politiques tout en prenant grand soin d'en informer la presse de l'époque dont la croissance rapide permit de lui offrir une publicité sans précédent. Après avoir terminé le mandat de William L. McKinley, assassiné, Roosevelt obtint la nomination du parti républicain pour être finalement élu président et servir son pays jusqu’en 1909, initiant ainsi un modèle que suivirent les quatre vice-présidents successeurs de présidents décédés ou démissionnaires : Coolidge (1924), Truman (1948), Johnson (1964) et Ford (1976). Seul ce dernier perdit ensuite l'élection. En servant de tremplin vers la présidence, la vice-présidence devint plus attractive pour des hommes de talent, tels Charles Dawes (1925-1929) prix Nobel, Charles Curtis (1929-1933) leader de la majorité au Sénat ou John N. Garner (1933-1941) «Speaker» de la Chambre des Représentants.

Malgré ces progrès, le vice-président restait néanmoins un homme de seconde main, que le président ne tenait pas toujours suffisamment informé. Ainsi, la mort de Franklin Roosevelt en 1945 créa une situation embarrassante où l'ancien sénateur du Missouri Harry Truman se vit propulsé à la tête de la nation. Son manque d'enthousiasme et surtout de préparation à cette succession força les responsables politiques de l'époque à reconsidérer la vice-présidence. Lavènement de la Guerre Froide avec l'Union Soviétique et la prolifération des

6 Nelson Michael, Guide to the Presidency (Thousand Oaks, CA: Congressional Quarterly Press, 2015), p. 165.

7 Ibid.

8 In Handlin Amy (éd.), Dirty Deals, vol. 2 « Articles » (Santa Barbara, Cal.: ABC-CLIO, LLC, 2014), p. 415-416. 
missiles nucléaires contribuèrent encore un peu plus à cette prise de conscience au sein de la classe politique et de l'opinion publique. En conséquence, la relation entre les deux hommes de l'Exécutif se fit plus étroite, le vice-président étant tenu mieux informé et jouant un rôle plus actif. Par exemple, en 1949, il fut intégré comme membre officiel au " National Security Council ».? Le président Dwight Eisenhower fut un grand partisan de cette évolution et déclara solennellement à propos de la mission de Richard Nixon : «I personally believe the Vice President of the United States should never be a nonentity. I believe he should be used. I believe he should have a very useful job. ${ }^{10}$

Avec la période d'après-guerre, une attention accrue fut portée à la compétence et à la loyauté des candidats à la vice-présidence. Certes, le colistier était toujours choisi en fonction de sa capacité à faire gagner des voix au ticket mais la sélection se fondait aussi sur la bonne connaissance des dossiers et la confiance mutuelle. Ce fut particulièrement le cas avec l'expérimenté Dick Cheney (2001-2009), ancien Ministre de la Défense (1989-1993) et ancien chef de cabinet de la Maison Blanche (1975-1977) qui fut choisi par George W. Bush et jouera ensuite un rôle prépondarant. Comme le rappelle James Pfiffner : "Cheney was chosen for his experience, competence, and his relationship with the head of the ticket. ${ }^{11}$

Le vingt-cinquième amendement de la Constitution, adopté le 23 février 1967, définit aussi plus clairement la fonction. Dans sa Section 1, il révisa le libellé controversé et ambigu de l'article II (Section 1, Clause 6) de la Constitution qui ne précisait pas explicitement si le vice-président devenait " president » ou " acting president » en cas de décès ou de départ du président. Le nouveau texte, dans la Section 1, indiqua précisément : "In case of the removal of the President from office or of his death or resignation, the Vice President shall become President. ${ }^{12}$ Cet amendement créa aussi une procédure pour sélectionner un vice-président dans deux circonstances exceptionnelles : la vacance vice-présidentielle et l'incapacité du président à exercer ses fonctions. Précédemment, la vice-présidence avait connu la vacance à seize reprises durant les trente-six administrations de l'histoire, amenant les vice-présidents à la présidence sans être toujours remplacés dans la fonction qu'ils abandonnaient. Désormais, dans sa Section 2, le texte stipule : "Whenever there is a vacancy in the office of the Vice President, the President shall nominate a Vice President who shall take office upon confirmation by a majority vote

9 Kengor Paul, Wreath Layer or Policy Player? The Vice President's Role in Foreign Policy. Lanham, MD: Lexington Books, 2000, p. 24.

10 Ibid., p. 43.

11 Pfiffner James P., The Modern Presidency (Belmont, CA: Thomson Wadworth, 2008), p. 90.

12 "The Constitution of the United States of America ", Amendment XXV. <http://constitutioncenter.org/constitution/the-amendments/amendment-25-presidential-disability-and-succession> Consulté le 13 juin 2015. 
of both Houses of Congress. ${ }^{13}$ Cette procédure fut mise en application en 1973 dans des circonstances inattendues où Spiro Agnew, impliqué dans une affaire de corruption, dut démissionner et laisser son poste à Gerald Ford. Quelques mois plus tard, la démission de Richard Nixon entraîna sa succession par Gerald Ford et le remplacement de celui-ci à la vice-présidence par Nelson A. Rockefeller. Le nouveau texte sur l'incapacité du président permit aussi de mettre fin au flou du libellé initial (article II, Section 1, paragraphe 6). Comme l'indique Pierre Lagayette :

"The Constitution did not say what a disability was, how the decision that the vice-president should step in would be made, or even whether the vicepresident would become president for the remainder of the four-year term or merely assume temporarily the powers and duties of the office. $»^{14}$

Cette imprécision fut particulièrement néfaste lors des présidences Garfield et Wilson. Dans le premier cas, la nation fut privée de président pendant près de trois mois ${ }^{15}$ et en septembre 1919, Wilson subit une attaque cérébrale qui le laissa paralysé et très affaibli sans toutefois abandonner le pouvoir. Les Sections 3 et 4 du nouvel amendement levèrent les ambiguités. La Section 3 précise d'abord : "Whenever the President transmits (...) his written declaration that he is unable to discharge the powers and duties of his office, (...) such powers and duties shall be discharged by the Vice President as Acting President. » La Section 4 ajoute : "Whenever the Vice President and a majority of either the principal officers of the executive departments or of such other body as Congress may by law provide, transmit (...) their written declaration that the President is unable to discharge the powers and duties of his office, the Vice President shall immediately assume the powers and duties of the office as Acting President. ${ }^{16}$

Investi d'un pouvoir exécutif plus clairement défini, assumant des responsabilités toujours plus importantes, le vice-président est donc devenu progressivement le second personnage le plus puissant de Washington, occupant " the second-highest office in the land. " ${ }^{17}$ Ce positionnement a fait des vice-présidents des candidats crédibles à la nomination de leur parti aux présidentielles, huit d'entre eux l'ayant obtenue sur les douze dernières administrations : Nixon (1960), Humphrey (1968), Mondale (1984), Bush (1988), Gore (2000) et les trois présidents successeurs mentionnés plus haut :

13 Ibid.

14 Lagayette Pierre, L'Empire de l'Exécutif (1933-2006) - La présidence des États-Unis de Franklin D. Roosevelt à George W. Bush (Paris: Presses de la Sorbonne, 2007), p. 76.

15 Garfield, victime d'un attentat, n'était pas en mesure d'assumer ses fonctions et le viceprésident Arthur refusa de tenir le rôle de président alors que Garfield était encore en vie.

16 "The Constitution of the United States of America ", Amendment XXV. <http://constitutioncenter.org/constitution/the-amendments/amendment-25-presidential-disability-and-succession> Consulté le 13 juin 2015.

17 Raphael, op. cit., p. 121. 
Truman, Johnson et Ford. Cette position favorable est aussi la conséquence du XXII ${ }^{e}$ amendement (1951) limitant la présidence à deux mandats. Le viceprésident est ainsi en mesure de se présenter aux élections durant le second mandat de son président sans gêner celui-ci. Ce fut le cas de Nixon en 1960, Bush en 1988 et Gore en 2000. Certes, obtenir une nomination ne signifie pas gagner une élection comme le vécurent amèrement Nixon et Gore, si courtes furent leurs défaites. Il n'en demeure pas moins que comme le rappelle Michael Nelson, "no office provides a likelier passage to the presidency than the vice-presidency. $»^{18}$

\section{First Lady : premier rôle?}

L'histoire présidentielle, plus particulièrement au $\mathrm{XX}^{\mathrm{e}}$ siècle, montre aussi que parmi les "proches " conseillers du président, figure un personnage de première importance : son épouse, la First Lady. Non mentionnée dans la Constitution, non élue, non rémunérée, sans mandat officiel, elle est néanmoins devenue un élément-clé de l'administration présidentielle. Malgré son absence de statut, elle joue un rôle primordial, aussi bien pendant la campagne présidentielle, par son précieux soutien, qu'au cours du mandat de son mari. Que ce soit dans ses tâches traditionnelles ou dans son rôle de représentation, comme miroir et modèle de la classe féminine ou encore dans sa participation plus ou moins directe aux décisions présidentielles, sa fonction s'avère éminemment politique.

C'est durant la campagne de 1992 et les premières années du mandat de Bill Clinton que le peuple américain vit l'émergence d'une Première Dame fonctionnant, pour ainsi dire, comme membre à part entière du gouvernement. L'interview accordée par Bill Clinton à Time dès sa prise de fonction en janvier 1993 est assez révélatrice :

"QUESTION : John Kennedy said that after he was elected, he began to think in terms of who it was he had to have in the room when he made the really big decisions. For him, that was Robert Kennedy. Who is it for you?

ANSWER : Hillary. ${ }^{19}$

Le président fraîchement élu confirmait ainsi sa promesse de campagne selon laquelle il comptait bien solliciter son épouse dans la prise de décisions. Au-delà de leur effet tapageur, les slogans bien connus tels que "Buy one, Get one Free ", " two for the price of one " ${ }^{20}$, marquaient la volonté d'une

18 Nelson Michael, "The Vice Presidency ", in Nelson Michael (éd.), The Presidency-A history of the office of the President of the United States from 1789 to the present (London: Salamander Books, 1996), p. 41.

19 Muller Henry \& Stacks John F., «First we have to roll up our sleeves », Time, 4 janvier 1993, p. 27.

20 Voir notamment Burns Lisa M., First Ladies and the Fourth Estate: Press Framing of Presidential Wives (DeKalb, Ill.: Northern Illinois University Press, 2008), p. 140. 
implication politique de la Première Dame. Une telle ligne de conduite n'était pas nouvelle pour les Clinton puisque toute la carrière politique de "Bill " avait été marquée par l'empreinte "Hillary ". Depuis que Bill s'était lancé dans ses premières joutes électorales en 1978 lors de la course au poste de gouverneur de l'Arkansas, le couple avait toujours opéré comme un véritable composé politique que les médias surnommèrent "Billary ", terme péjoratif pour certains, admiratif pour d'autres. ${ }^{21}$

Cette administration bicéphale s'inscrivait dans le droit fil des ambitions carriéristes et complémentaires de ce couple hors du commun. Une nouvelle dynamique présidentielle avec redéfinition des postes se mit donc en place. À la différence de ses prédécesseurs, Mrs. Clinton investit l'Aile Ouest de la Maison Blanche, "with all the men. "22 Ce déménagement fut certainement un des signes les plus symboliques de l'ambition de Mrs. Clinton et des moyens qui lui étaient alloués pour parvenir à concrétiser cette ambition. «Hillary and her sixteen aides ", écrit l'historien Gil Troy, " established what would be known as "Hillaryland". This enclave included an unprecedented three aides honored with White House commissions; in contrast, the vice president's staff had one. $»^{23}$ La presse n'a pas manqué de relater cette rupture avec la tradition. Time le 8 février consacrait tout un article aux nouveaux appartements de la Première Dame, gratifiant même le lecteur d'un graphique très précis sur les affectations des différents bureaux de l'Aile Ouest, intitulé : « WHERE SHE WORKS ». ${ }^{24}$ Les titres de presse abondent pour illustrer le pouvoir de la Première Dame : «A Room at the Top ${ }^{25}$, , At the Center of POWER $»^{26}$, " the most controversial -and most powerful- First Lady in years $»^{27}$, "How much Clout? ${ }^{28}$, « Hillary Clinton's office, near seat of power, signals her influential role $»^{29}$, etc. Margaret Carlson écrivit alors : "At the moment, other than the President, there is no more powerful person in the White House than Hillary Clinton. " ${ }^{30}$

21 Caroli Betty Boyd, First Ladies: From Martha Washington to Michelle Obama (New York/ Oxford University Press, 2010), p. 294.

22 Carlson Margaret, "A Room at the Top ", Time, 8 février 1993, p. 31.

23 Troy Gil, Affairs of State-The Rise and Rejection of the Presidential Couple Since World War II (New York: The Free Press, 1997), p. 356.

24 Carlson, op. cit.

25 Titre de l'article de Margaret Carlson, ibid.

26 Carlson Margaret, "At the center of Power ", Time, 10 mai 1993, p. 38.

27 Sous-titre de l'article de Diana McLellan, "What Hillary's learned », Ladies Home Journal, août 1993, p. 118.

28 Couverture Newsweek, 15 février 1993.

29 Susan Baer, «Hillary Clinton's office, near seat of power, signals her influential role », Baltimore Sun, 22 janvier 1993. <http://articles.baltimoresun.com/1993-01-22/news/1993022176_1_ east-wing-west-wing-main-office> Consulté le 12 juillet 2015.

30 Carlson Margaret, "A Room at the Top », op. cit. 


\section{Confrontation}

Inévitablement, sont alors apparues des tensions dont la plus marquante émana du vice-président $\mathrm{Al}$ Gore qui, relégué au rôle de $\mathrm{n}^{\circ} 3$, n’apprécia guère un tel dispositif et perçut Hillary Clinton comme sa rivale directe en matière de pouvoir. Il est vrai que ce système exécutif à trois têtes avec un président, une First Lady et un vice-président marginalisait ce dernier, bien que $n^{\circ} 2$ officiel, et le privait de son rôle communément admis de "chief lieutenant " du président ${ }^{31}$. William A. Kristol, l'ancien chef de cabinet de Dan Quayle, le précédent vice-président de l'administration Bush, ne se priva pas de railler la situation peu confortable de Mr. Gore : «He [Gore]'s clearly not the second banana. He's the third banana, behind Mrs. Clinton. „ ${ }^{32} \mathrm{Al}$ Gore se voyait en effet éclipsé par sa rivale qui, elle, était scrutée en permanence par le public et les médias, nationaux et internationaux. Contrairement à ceux de la First Lady, les déplacements du vice-président recevaient une couverture médiatique plutôt discrète. En juillet 1993 par exemple, lors d'un voyage à Ontario, Californie, pourtant soigneusement programmé pour passer en " prime time » le soir à la télévision, aucune station locale ne jugea utile de couvrir la venue du vice-président. L'attitude des télévisions ${ }^{33}$ était significative du manque d'intérêt à l'égard du vice-président et mettait en lumière le malaise qui régnait à la Maison Blanche avec le rôle croissant d'une Première Dame déterminée et médiatisée face à un vice-président qui paraissait isolé et donc affaibli, contraint de s'accommoder d'un rôle subalterne peu compatible avec une conception moderne de sa fonction.

Et pourtant, Bill Clinton et $\mathrm{Al}$ Gore s'étaient préalablement mis d'accord sur les tâches et les missions qui incombaient au vice-président. En le choisissant comme " running mate ", le candidat Clinton l'avait présenté comme celui qui allait diriger l'action avec le Congrès pour faire passer le programme présidentiel dès les premiers mois de son administration : «I am going to send Al Gore to Capitol Hill to take the lead in passing our program in the first 100 days of the new administration. ${ }^{34}$ Le choix de Gore se démarquait du mode opératoire habituel selon lequel le candidat présidentiel choisit son colistier avec un souci

31 Williams Irving G. "Vice Presidency of the United States. »US Government. Expert Space, 2015. Web. 3 sept. 2015. <http://freedomflix.digital.scholastic.com/exploremore_ pdf/01000364/fflix/ngo//10011289> Consulté le 2 août 2015.

32 Nelson Michael, "Vice President Gore: Not Second Fiddle ", Baltimore Sun, 1 ${ }^{\text {er }}$ août 1993. <http://articles.baltimoresun.com/1993-08-01/news/1993213096_1_vice-presidency-gorepresident-in-history> Consulté le 12 juillet 2015.

33 West Paul, "Gore leaves few tracks, stays close to Clinton Profile contrasts to Dan Quayle's role ", The Baltimore Sun, 15 février 1993. <http://articles.baltimoresun.com/1993-02-15/ news/1993046127_1_al-gore-president-and-vice-president-ronald-reagan/2> Consulté le 12 juillet 2015.

34 <http://www.docstoc.com/docs/167288426/Bill-Clintons-Campaign-Promises> Consulté le 12 juillet 2015. 
d'équilibre du ticket, qu'il soit géographique, idéologique ou générationnel, susceptible de récolter le plus grand nombre de voix. Clinton adopta au contraire " the antithesis of the conventional models » rappelle Joel Goldstein, en sélectionnant " the one closest to being a carbon copy of himself. $~^{35}$ Gore présentait en effet de nombreuses similitudes avec Bill Clinton : sudiste (Tennessee), baby boomer (Gore est né en 1948 et Clinton en 1946), baptiste, démocrate modéré et fin politicien. Clinton avait choisi ce vice-président car il existait entre eux " a definite simpatico ". ${ }^{36}$ Avant d'entrer en fonction, les deux hommes avaient signé un document écrit déclinant les principales missions du vice-président en matière d'environnement, de politique étrangère, de sécurité, de réforme administrative, etc, de même que son rôle plus général de conseiller. ${ }^{37}$ Puis les deux hommes décidèrent ensuite, sous l'insistance d'Al Gore, qu'ils se rencontreraient chaque jeudi pour un déjeuner d'affaires privé. Gore avait le sentiment que si une bonne relation, personnelle et professionnelle, n'était pas entretenue, alors elle pouvait devenir "vulnerable. ${ }^{38}$

Ce sentiment était prémonitoire car l'influence du vice-président fut considérablement réduite par l'omniprésence d'Hillary Clinton. Le terme " co-presidency » fut utilisé dans plus de 92000 articles en 1993 ! ${ }^{39}$ Comme l'écrivit alors l'historien D. Herbert Lipson :

"It used to be that when we elected a president, the vice president was automatically tacked on as part of the package. Now, though, we've made a quantum leap. With Bill Clinton, we truly elected the First Couple (...). » ${ }^{40}$

Le couple travaillait en étroite collaboration et l'influence de la Première Dame dans la prise de décisions était à peine voilée. Le président, qui avait une confiance aveugle en son épouse pour son sens politique très affûté, se faisait même un devoir de travailler avec elle : "I would be derelict in my duty to the United States if I did not use her " déclara-t-il. ${ }^{41} \mathrm{Il}$ en était à la présidence comme par le passé. Le modus operandi restait le même. "Hillary had an obvious advantage over Gore ", écrit la biographe Sally Bedell Smith, "because she and Bill had been on the same wavelength for so long that they communicated almost by telepathy. " ${ }^{42}$

35 Goldstein Joel K., "Clinton, Gore and the New Vice Presidency. " In Perotti Rosanna (éd.), The Clinton Presidency and the Constitutional System (College Station, TX: ATM, 2012), p. 87.

36 Pfiffner, op. cit., p. 86.

37 Ibid.

38 Smith Sally Bedell, For Love of Politics - Inside the Clinton White House (New York: Random House, 2008), p. 93.

39 Troy, op. cit., p. 355.

40 Lipson D. Herbert, "Off the Cuff ", Philadelphia magazine, vol. 84, n 11, novembre 1993 , p. 1.

41 Walsh Kenneth T. \& Toch Thomas, "Now the First Chief Advocate ", US News \& World Report, 25 janvier 1993, p. 46.

42 For love of Politics, op. cit., p. 92. 
Cependant, le rôle de "partenaire " exercé par une personne non élue ne peut évidemment pas être épargné par la critique, particulièrement s’il s'agit d'une femme. Comme le montrent de nombreux exemples dans l'histoire, derrière les critiques à l'égard des femmes de présidents se cache la crainte d'un " petticoat government " censé menacer le pouvoir masculin. ${ }^{43}$ Abigail Adams, fervent défenseur de la cause des femmes, ne cessa de maintenir un partenariat politique avec John son mari, ce qui l'amena à être affublée, comme Rosalynn Carter et Hillary Clinton, du surnom de "Mrs. President " ou comme Eleanor Roosevelt de "minister without portfolio ». ${ }^{44}$ Sous l'administration Polk (1845-1849) le vice-président George M. Dallas se demanda si Sarah, la femme du président, était le second dirigeant politique le plus important de la nation. "She is certainly mistress of Herself ", nota-t-il avec sarcasme, "and I suspect of somebody else also. " ${ }^{45}$ Eleanor Roosevelt fut aussi violemment critiquée pour son implication dans l'administration de FDR dont elle était « the eyes and ears. $»^{46}$ Un journaliste espagnol la traita de " macherras » et posa la question : "Is Mrs. Roosevelt a sort of Stalin in petticoats? Where does her power lie? ${ }^{47}$ Le chef de cabinet de la Maison Blanche sous Ronald Reagan, Donald Regan, s'en prit vivement à la Première Dame dans ses mémoires : «Mrs. Reagan regarded herself as the president's alter ego not only in the conjugal but also in the political and official dimensions, as if the office that had been bestowed on her husband by the people somehow fell into the category of worldly goods covered by the marriage vows. " ${ }^{48}$ Le rôle très actif de Rosalynn Carter, la première First Lady à assister aux réunions de cabinet, amena un journal à titrer «Is Rosalynn really running the country? "

S'il y a bien eu d'autres Premières Dames influentes avant elle, c'est néanmoins Hillary Clinton qui a exercé cette influence le plus ouvertement dans l'histoire moderne. "Never before has a woman been the power behind the throne so much as Hillary Rodham Clinton " écrit Gay Smith. ${ }^{50}$ Pendant la campagne, Mrs. Clinton avait parfois été présentée comme " the president's running mate " empruntant une expression qui désigne normalement le

43 Weisberger Bernard A., "Petticoat Government ", American Heritage, octobre 1993, p. 20.

44 Ibid., p. 18.

45 Sferrazza Anthony Carl, First Ladies - The Saga of the Presidents' Wives and their Power 17891961, vol. 1 (New York: William Morrow, 1990), p. 140.

46 Voir par exemple DuBois Ellen Carol, Through Women Eyes (New York: Bedford-St Martin's, 2012), p. 566.

47 In Lash Joseph P., Eleanor - The Years Alone (New York: Norton \& Co., 2014), p. 307.

48 Regan Donald, For the Record (New York: Harcourt Brace Jovanovich, 1988), p. 288.

49 In Kaufman Burton I. \& Kauffman Scott, The Presidency of James Earl Carter, Jr. (Lawrence, KS :University Press of Kansas, 2006), p. 214.

50 Smith Gay, Lady Macbeth in America - From the Stage to the White House (New York: Palgrave Macmillan, 2010), p. 15. 
candidat à la vice-présidence ! $!^{51}$ À la Maison Blanche, il fallait se faire à l'idée qu' "Hillary along with Bill and Gore had to "sign off on big decisions" " confie le chef de cabinet Mack McLarty. ${ }^{52}$ Pour Al Gore, cette mise en avant de la Première Dame, non élue, équivalait à une forme d'usurpation de pouvoir. Lépisode le plus emblématique et le plus conflictuel fut la décision présidentielle de confier à la Première Dame la mise en place de la réforme du système de Santé, étape voulue comme marquante du premier mandat de Bill Clinton, et qu'Al Gore avait lui-même convoitée en raison de ses compétences législatives acquises en tant que Sénateur des États-Unis. C'est pourtant la First lady qui eut les faveurs du président. «Bill was investing Gore with considerable responsibility, but his failure to confide in his vice-president was a telling sign of the real pecking order " explique Sally Smith. ${ }^{53}$ Son choix était lié à des raisons de personnalité plus que de compétences. Il avait besoin d'une personne médiatisée et très en vue, capable de " vendre » la réforme et gagner les soutiens nécessaires pour faire passer la loi au Congrès. La First Lady était pour lui la personne idéale. Infatigable et persuasive, elle allait parcourir le pays, tenir des audiences publiques et vanter inlassablement la réforme. Bien que bon orateur, le viceprésident avait toujours selon Bill Clinton, une apparence un peu trop rigide, le ton moralisateur, et une tendance compulsive à l'abstraction dans son discours. ${ }^{54}$ Or, la réforme de la Santé allait toucher les gens au plus près, et imposait donc un message plus " populaire " dans sa forme, message que la Première Dame était, toujours selon Bill Clinton, plus à même de faire passer.

La culture populaire se fit le témoin du décalage entre le poids politique de Mrs. Clinton et celui d'Al Gore. En 1993, par exemple, les stations de radio diffusèrent une parodie de la chanson de Helen Reddy, "I Am Woman »: «I am Hillary, hear me roar, I'm more important than $\mathrm{Al}$ Gore $» .^{55} \mathrm{Au}$-delà de la parodie, on voit que Mrs. Clinton donnait une nouvelle dimension au statut de First Lady. Comme l'explique l'auteur Robert P. Watson :

«It is becoming the rule and not the exception that the first lady has surpassed the vice president and even the most senior advisers and cabinet secretaries in terms of visibility and perhaps even power and influence both in and out of the White House. " ${ }^{56}$

51 Voir par exemple Meyer Karl E., "The President's Other Running Mate », The New York Times, 27 janvier 1993, p. A 12.

52 In For Love of Politics, op. cit., p. 91-92.

53 Ibid., p. 81.

54 Ibid., p. 211.

55 Voir notamment Green Michelle, "Her own woman ", People Weekly, 10 mai 1993, p. 83.

56 Watson Robert P., "The First Lady Reconsidered: Presidential Partner and Political Institution ", Presidential Studies Quarterly, vol. 27, n 4, automne 1997, p. 814. 
Cet ascendant politique de la Première Dame résultait aussi du fait que l'image de candeur et d'honnêteté qui caractérisait $\mathrm{Al} \mathrm{Gore}{ }^{57}$ et qui avait été fort utile pendant la campagne car elle avait permis de contrebalancer celle d'incorrigible "womanizer » de Bill Clinton, surnommé alors "Slick Willie " ${ }^{58}$, n'avait plus beaucoup d'impact maintenant que la Maison Blanche était conquise. ${ }^{59}$ Une autre hypothèse fut que Bill Clinton voulait compenser le tort qu'il faisait à son épouse par ses infidélités conjugales en lui accordant un pouvoir qu'elle n'aurait légitimement pas dû avoir, aux dépens du vice-président. Ce sentiment réapparut en 1998, quand Hillary se présenta au Sénat de New York. "Clinton was eager for Hillary to win the Senate race to recompense her for all she had to put up with " raconte Madeleine Albright. ${ }^{60}$ Enfin, son rôle croissant en tant que First Lady n'était perçu que comme la première étape stratégique d'une ascension programmée vers la présidence. ${ }^{61}$

Comme le soulignent les chroniqueurs Karen Tumulty et Nancy Gibbs, «Bill et Hillary » ont sans doute formé le plus politique des couples présidentiels américains :

"The history of the Clinton presidency is and always has been the history of the Clinton marriage, which is why the distinction between public and private in this presidency has always been messy. From the start their union was a vessel not only of love but of ambition, a shortcut for two stars in a hurry to reach heaven (...). And there was always the risk that if one stumbled, it would bring down the other too. " ${ }^{62}$

La suite a en partie donné raison à cette prédiction. Le couple Clinton fut vite fragilisé par les révélations répétées des infidélités conjugales du mariprésident, lesquelles ont eu l'effet pernicieux d'auréoler les deux époux, à l'origine présentés comme un duo indéfectible, d'un halo d'imposture. Leur image de marque, qui mêlait habilement et harmonieusement leurs relations publique et privée, au service d'une présidence exemplaire, se vida de son sens et vint anéantir l'authenticité du message d'union parfaite. De plus, après l'échec de la réforme de la Santé et les sondages défavorables, la co-présidence fut vite abandonnée et la Première Dame contrainte et forcée par la machine présidentielle de rester en retrait et de tenir un rôle purement traditionnel. ${ }^{63}$

57 Gore fut d'ailleurs affublé des surnoms de «Mr. Clean », « Mr. Faithful » ou encore « DudleyDo-Right » personnage de dessin animé un peu naïf et ingénu. In Smith, ibid., p. 92.

58 Voir notamment sur l'affaire "Gennifer Flowers »: Maraniss David, First in His Class (New York: Simon \& Schuster, 1996), p. 354.

59 For Love of Politics, op. cit., p. 92.

60 Ibid., p. 374.

61 Ibid., p. 92, p. 488.

62 Tumulty Karen \& Gibbs Nancy, " Hillary Clinton:The Better Half - During her husband's greatest crisis, Hillary has come into her own ", Time, 28 décembre 1998. <http://content. time.com/time/magazine/article/0,9171,989909,00.html> Consulté le 17 juin 2015.

63 Cf. James Bowman, "The incredible Shrinking Lady ", National Review, 18 avril 1994, p. 24. 
Quant à $\mathrm{Al}$ Gore, selon le principe des vases communicants, il retrouva un rôle de vice-président plus estimable comme " general adviser to the president" tout en endossant les tâches exécutives afférentes selon les besoins : présidence du Sénat, politique étrangère, réorganisation des agences gouvernementales, etc. Les relations entre le président et le vice-président s'améliorèrent au fil des semaines et retrouvèrent une certaine forme de normalité, Clinton appréciant la clarté des analyses et les qualités d'organisation de son vice-président, Gore faisant preuve de loyauté à l'égard de son président, recherchant le consensus et apportant une aide précieuse dans la prise de décision. ${ }^{64}$ Emboîtant le pas à ses prédécesseurs, il consolida la fonction en contribuant ainsi à faire de la vice-présidence une institution moderne et efficace, " a leap forward for that often-pilloried institution. ${ }^{65}$ Plus tard, avec du recul, Clinton dira même de lui qu'il fut " the single most influential, effective, powerful, and important vice president in the history of the United States of America. " ${ }^{66}$

Pourtant, la rivalité entre Gore et Hillary Clinton ressurgit nettement en 2000 lorsque les deux protagonistes entrèrent en campagnes, l'un pour la présidence, l'autre pour le Sénat de New York. Ce télescopage du calendrier raviva les vieilles rancours du pouvoir tripartite des premières années, alors décrit comme "a madhouse $»^{67}$ : un président canard boiteux rongé par les scandales, une épouse trahie mais déterminée à se faire élire et un successeur désigné de Martin Van Buren et George Bush, Sr. Novembre mit fin aux ambitions présidentielles de Gore, dont la campagne souffrit en partie de l'affaire Lewinsky, dégradante pour le pays, déclencheur de sentiments négatifs : animosité envers Bill Clinton, lassitude à l'égard du couple Clinton, et volonté d'en finir avec l'administration Clinton ${ }^{68}$. Au grand bonheur de George W. Bush.

\section{Conclusion}

L'historique des deux mandats montre que la confusion ayant régné à la Maison Blanche s'est manifestée à plusieurs niveaux. Dans un premier temps, la « co-présidence " Clinton relégua le vice-président au rôle paradoxal et subalterne de simple sous-fifre, " underling " au sein de l'organigramme présidentiel. Pour recouvrer du crédit électoral, le président fut ensuite contraint

64 In For Love of Politics, op. cit., p. 92-93.

65 Goldstein, op.cit., p. 86.

66 "Remarks at a Gore 2000 Meeting in Little Rock, Arkansas, August 7, 1999. » Administration of William J. Clinton, 1999. Public Papers of the Presidents of the United States, 1403.

67 Bose Meena, "Reclaiming the "Vital Center" in American Politics through the Clinton Presidency ". In Perotti, op. cit., p. 5.

68 Ces sentiments furent regroupés sous l'appellation «Clinton fatigue». In Dover Edwin, Missed Opportunity - Gore, Incumbency and Television in Election 2000 (New York: Greenwood Press, 2002), p. 130. 
de céder à la tradition en neutralisant politiquement la Première Dame et en collaborant plus étroitement avec le vice-président, lequel retrouva ses galons de " chief lieutenant. » Ce retournement de situation fut complété par une troisième étape tumultueuse, l'affaire Lewinsky, qui vint déshonorer la présidence Clinton et créer un malaise rarement égalé dans l'histoire. Elle mit en scène un "Maître " plus vraiment maître de la situation car empêtré dans une procédure de destitution (" impeachment »), une First Lady trompée, éplorée et amère, et un vice-président qui bien qu'ayant regagné du crédit, se retrouvait malgré lui fragilisé par son appartenance au clan Clinton et au spectacle pitoyable qu'il offrait à son peuple.

Certes, le bilan d'une administration ne se réduit pas aux frasques sexuelles de son président ou à la rivalité de pouvoir entre une First Lady et un viceprésident. Cependant, la Maison Blanche Clinton a bel et bien été le théâtre d'une véritable comédie humaine, qui eût pu prêter à sourire si elle n’avait provoqué un désaveu général et une décrédibilisation d'un pouvoir que ses détenteurs étaient, par définition, censés incarner. David Gergen, éminent commentateur politique, conseiller des présidents Nixon, Ford, Reagan, et Clinton, ne fut pas tendre avec les protagonistes de ce triumvirat qu'il qualifia sans détour de " rolling disaster. " ${ }^{69}$

\author{
Pierre-Marie Loizeau \\ EA 4335 3L.AM \\ Université d'Angers \\ pm.loizeau@yahoo.fr
}

\title{
Résumé
}

Cet article s'attache à analyser la rivalité entre Hillary Clinton et Al Gore durant la présidence Clinton. Les réformes successives de la fonction de vice-président ont permis à celui-ci de tenir progressivement le rang de $\mathrm{n}^{\circ} 2$ au sein de l'Exécutif. En inversant la tendance au profit de la Première Dame, l'administration Clinton instaura une rivalité de pouvoir entre seconds couteaux. L'échec de la réforme du Système de Santé écarta Mrs. Clinton et rétablit une collaboration plus traditionnelle entre le président et le vice-président. En fin de mandat, le scandale Lewinsky entraîna le discrédit de toute l'administration y compris du vice-président et de la Première Dame.

\section{Mots-clés}

Président des États-Unis, Vice-Président des États-Unis, Première Dame, Hillary Clinton, AL Gore.

69 Gergen David, Eyewitness to Power: The Essence of Leadership, Nixon to Clinton (New York: Simon \& Schuster, 2000), p. 293. 


\section{Abstract}

This paper explores the struggle for power between Hillary Clinton and Al Gore during the Clinton presidency. Historically, the successive reforms of the vice presidency have gradually made it the second-highest office in the land. The trend was reversed as the Clinton administration gave political prominence to the First Lady, thereby antagonizing the two sidekicks. The failure of the Healthcare reform forced Mrs. Clinton aside and restored a more traditional collaboration between the president and the vice president. The end of the Clinton era was marked by the Lewinsky scandal and the ensuing discredit on the whole administration including the vice president and the First Lady.

\section{Keywords}

United States President, United States Vice-President, First Lady, Hillary Clinton, AL Gore. 